\title{
Recent evidence on modifiable and non-modifiable risk factors for colorectal cancer (CRC): a systematic synopsis of meta-analyses from 2015 to 2017
}

Teguh Kristian Perdamaian ${ }^{1,2}$

pISSN: 0853-1773 • elSSN: 2252-8083 https://doi.org/10.13181/mji.v28i2.2679 Med J Indones. 2019;28:188-95

Received: July 27, 2018

Accepted: March 31, 2019

\section{Author's affiliations:}

${ }^{1}$ Faculty of Medicine, Universitas Kristen Duta Wacana, Yogyakarta, Indonesia ${ }^{2}$ Centre of Population Health Sciences, The University of Edinburgh, Edinburgh, United Kingdom

\section{Corresponding author:} Teguh Kristian Perdamaian Faculty of Medicine, Universitas Kristen Duta Wacana, Gedung Logos, Jalan Dr. Wahidin Sudirohusodo No. 5-25, Yogyakarta 55224, Indonesia Tel/Fax: +62-274-563929

E-mail: teguh.kristian@staff.ukdw.ac.id

\begin{abstract}
Colorectal cancer (CRC) is a common cancer with a huge impact on international public health. This review discusses recent evidence on modifiable and non-modifiable risk factors for $\mathrm{CRC}$ using a systematic review method. This systematic review was conducted according to the Preferred Reporting Items for Systematic Reviews and Meta-Analyses guidelines on systematic reviews and meta-analyses of observational studies. The literature search was performed on the Ovid MEDLINE database and included publications from 2015 to 2017 , followed by a quality assessment and a narrative synthesis. Of the 90 identified articles, there were 13 meta-analyses with statistically significant results. Seven articles discussed modifiable risk factors and six articles discussed non-modifiable risk. The modifiable risk factors with the highest risk were radiotherapy of prostate cancer (pooled odds ratio 1.68; 95\% confidence interval $[\mathrm{Cl}]$ 1.33-2.12). The non-modifiable risk factors with the highest risk was Lynch syndrome (hazard ratio 135.49; 95\% Cl 111.55-164.57). This review discovered new and previously known risk factors for CRC. Recent evidence shows that research on CRC risk factors is continuing to grow indicating that more studies on risk factors are needed to optimize CRC prevention and early detection.
\end{abstract}

KEYWORDS colorectal cancer, genetic marker, metabolic diseases, meta-analysis, risk factors
Colorectal cancer (CRC) is a common cancer with a huge impact on public health worldwide. This cancer contributes to a large incidence and mortality second only to breast cancer. Evidance show higher incidence (>14.3 per 1,000) and mortality rates (>7.7 per 1,000) in Europe, North America, and the Western Pacific region. However, recent studies have reported an increasing trend of incidence and mortality in developing countries such as in Southeast Asia and South America., Data from the global burden of disease (GBD) study showed similar results for middleincome countries, with up to a 3 -fold increase in CRC rate over 25 years. 3 Advances in communicable disease management in these countries might prolong life expectancy and expose the population to more risk factors for $C R C$ over their lifetime. Apart from geographical variability, CRC epidemiology appears to be influenced by socioeconomic inequalities, in which the highest deprived populations have the highest CRC incidence and mortality rates. ${ }^{4}$ 
Many reviews and meta-analyses have summarized important non-modifiable and modifiable risk factors for developing CRC. The 2015 GBD study reported that $52.57 \%$ of the colorectal cancer burden is attributable to behavioral and metabolic risk factors, including physical inactivity, a unhealthy diet, smoking, and obesity. ${ }^{1}$ Some preexisting diseases have also been reported to have a substantial risk on the development of CRC, such as hepatobiliary autoimmune disorders (i.e., primary sclerosing cholangitis) and inflammatory bowel diseases (i.e., ulcerative colitis). ${ }^{5}$ Another growing area of $C R C$ risk profiling is genetic studies, which have discovered several potential genetic polymorphisms that might be beneficial for CRC screening. For example, patients with Lynch syndrome and a mutation in the MSH1, MLH2, MSH6, or PMS2 genes are offered earlier colonoscopy screenings due to the high rate of future $C R C$ development. 6,7 Yet, there could be more undiscovered risk factors, which might improve current prevention practices. This review will discuss recent evidence on modifiable and non-modifiable risk factors to complement existing knowledge, by systematically searching and summarizing the current best evidence. The method used in this review will guide future health practitioners when they conduct concise systematic reviews on meta-analyses with a broad topic. The results from this review will be useful as a guide to direct future research or practice recommendations.

\section{METHODS}

\section{Search strategy and eligibility criteria}

A systematic literature search was conducted to identify meta-analysis studies that summarized risk factors for developing colorectal cancer. The risk factors were categorized into two main groups of modifiable and non-modifiable. The risks were not limited to one with evidence on causality, but we also considered other conditions related to the development of CRC. A modifiable risk factor was considered when there was any evidence of reduced risk from an intervention. Non-modifiable risk factors generally include aging, gender, ethnicity, and genetic characteristics. ${ }^{8}$ The outcome of this study was the pooled risk of certain risk factors on the outcome of all types of CRC. The pooled risk could be a risk ratio ( $R R$ ), odds ratio (OR), risk difference, or hazard ratio (HR), and should be complemented with its $p$-value and $95 \%$ confidence interval $(\mathrm{Cl})$. The types of CRC included in this study were colon cancer, rectal cancer, cecal cancer, sigmoid cancer, and hereditary nonpolyposis cancer. The search was conducted on the MEDLINE platform, using the subject headings, text words, and limitation features shown in Table 1.

No age, sex, or language limitations were followed. The search was limited by publication dates between January 1, 2015 and April 7, 2017. The search method for both types of risk factors was combined into one flow (Figure 1), in which the classification was developed after the full-text data were obtained. Based on the objective of this review, the literature search and screening included studies on humans, and the CRC diagnosis as the outcome. The type of study was restricted to a meta-analysis. This review excluded protective risk factors.

\section{Data extraction, quality assessment, and data synthesis}

Key information was extracted from the articles, and the results are presented in Table 2 and 3. The data included were authors, journal reference, studied risk factors and subsets, studies included (sample size, case and control number, association estimation (type of estimation [HR and RR], or OR and $95 \% \mathrm{Cl}$, and corresponding $p$-value), heterogeneity of the studies

Table 1. Search strategy in the MEDLINE platform

\section{Search terms}

(exp cecal neoplasms/or exp appendiceal neoplasms/or exp colorectal neoplasms/or exp adenomatous polyposis coli/ or exp gardner syndrome/ or exp colonic neoplasms/or exp sigmoid neoplasms/ or exp colorectal neoplasms, hereditary nonpolyposis/ or exp rectal neoplasms/) or ((cecal neoplasms or appendiceal neoplasms or colorectal neoplasms or adenomatous polyposis coli or gardner syndrome or colonic neoplasms or sigmoid neoplasms or colorectal neoplasms or hereditary nonpolyposis or rectal neoplasms).tw)

(exp risk/ or exp risk assessment/ or exp risk factors/) or ((risk or risk assessment or risk factors).tw.)

limit 3 to (humans and meta-analysis and "causation-etiology (maximizes specificity)" and yr="2015-Current") 

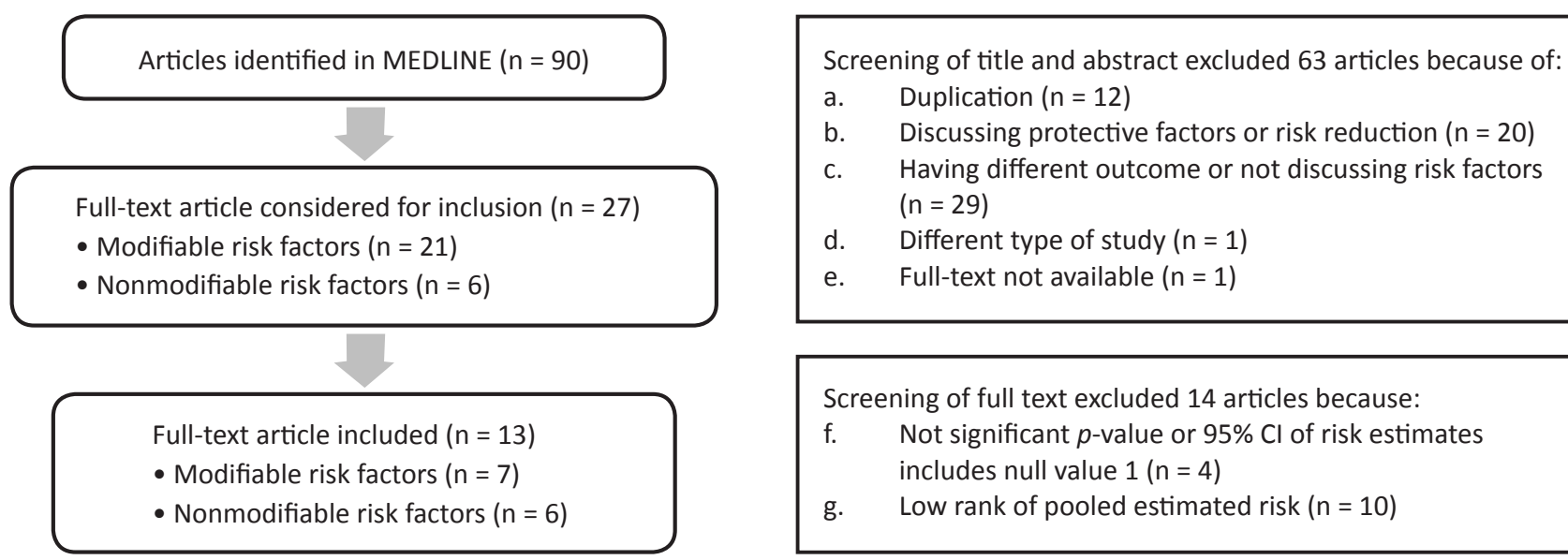

Figure 1. Flow diagram for the literature search and screening process

( $I^{2}$ and $p$-value), and quality of the meta-analysis. The trend or dose-response analysis was not inputted into the table or with the subgroup analysis and metaregression results. The values of implicit information were calculated from published data on the included meta-analyses and their primary sources when possible. The quality of the meta-analysis was assessed with Preferred Reporting Items for Systematic Reviews and Meta-Analyses (PRISMA) guidelines on systematic review and meta-analysis of observational studies reporting. ${ }^{9}$

\section{RESULTS}

Ninety articles were identified by the initial search strategy. Sixty-three papers were excluded due to failed eligibility criteria. Of the 27 studies remaining, 13 articles were included based on the focus on five risk factors in each group (Figure 1). These 10 risk factors were chosen based on their high rank on the magnitude of pooled risk estimates. Seven articles discussed five modifiable risk factors, such as abdominal radiation for other malignancies, ${ }^{10}$ alcohol and beer consumption, ${ }^{11,12}$ diabetes mellitus (DM), ${ }^{13,14}$ Helicobacter pylori infection, ${ }^{15}$ and gynecologic surgery. ${ }^{13}$ The other six articles discussed non-modifiable genetic factors, including Lynch syndrome (polymorphisms in the MLH1 and $\mathrm{MSH} 2$ genes), ${ }^{16,17}$ the rs16892766 polymorphism, ${ }^{18}$ the rs4779584 polymorphism, ${ }^{19}$ the XRCC1 gene polymorphism, ${ }^{20}$ and the BMP4 gene polymorphism. ${ }^{21}$ These studies are presented in Table 2 and 3 ordered by the earliest publication date. The publication quality of the seven included articles was exceptional, except for the articles by Guraya ${ }^{14}$ and Jenkins et $\mathrm{al}^{16}$ because of unavailable details on robust search methods and a bias assessment. As expected from meta-analyses of observational studies, heterogeneity between studies was a common finding ( 8 of 18 results had substantial heterogeneity with $\mathrm{I}^{2}>50 \%$ ).

\section{Modifiable risk factors}

Some considerably increased risks were reported for abdominal radiation exposure (OR 1.68; $95 \% \mathrm{Cl} 1.33-$ 2.12) and lifetime alcohol consumption (RR 1.49; $95 \% \mathrm{Cl}$ 1.27-1.74). Specifically, Zhang and Zhong ${ }^{12}$ reported a significant association between the alcohol in beer and the incidence of CRC (RR 1.20; 95\% Cl 1.06-1.37). There was a $21 \%$ increased risk $(95 \% \mathrm{Cl} 2-42 \%)$ of developing CRC in patients with type 2 diabetes mellitus (T2DM). ${ }^{14}$ In addition, Luo et al $^{22}$ reported a $37 \%$ increased risk for diabetics, which was not limited to T2DM. A review by Zhao et al ${ }^{15}$ suggested that a $\mathrm{H}$. pylori infection can also play a role in the development of CRC (RR 1.33; 95\% Cl 1.01-1.77), and is not limited to upper gastrointestinal cancer as previously thought. An increased risk (22-30\%) of CRC incidence was found in postsurgical women who had their ovaries removed (i.e., oophorectomy and hysterectomy). (Table 2)

\section{Non-modifiable risk factors}

Six of the included meta-analyses discussed genetic factors as non-modifiable risk factors. A substantial association was detected between a genetic mutation related to Lynch syndrome and one type of CRC (hereditary nonpolyposis colorectal cancer) (HR 135.49; 95\% Cl 111.55-164.57). MLH1 also showed significant increase in odds of developing all types of CRC (codominant polymorphism, OR 2.29; $95 \% \mathrm{Cl}$ 1.618-3.244). Two major single nucleotide polymorphisms (SNPs) had considerably increased 


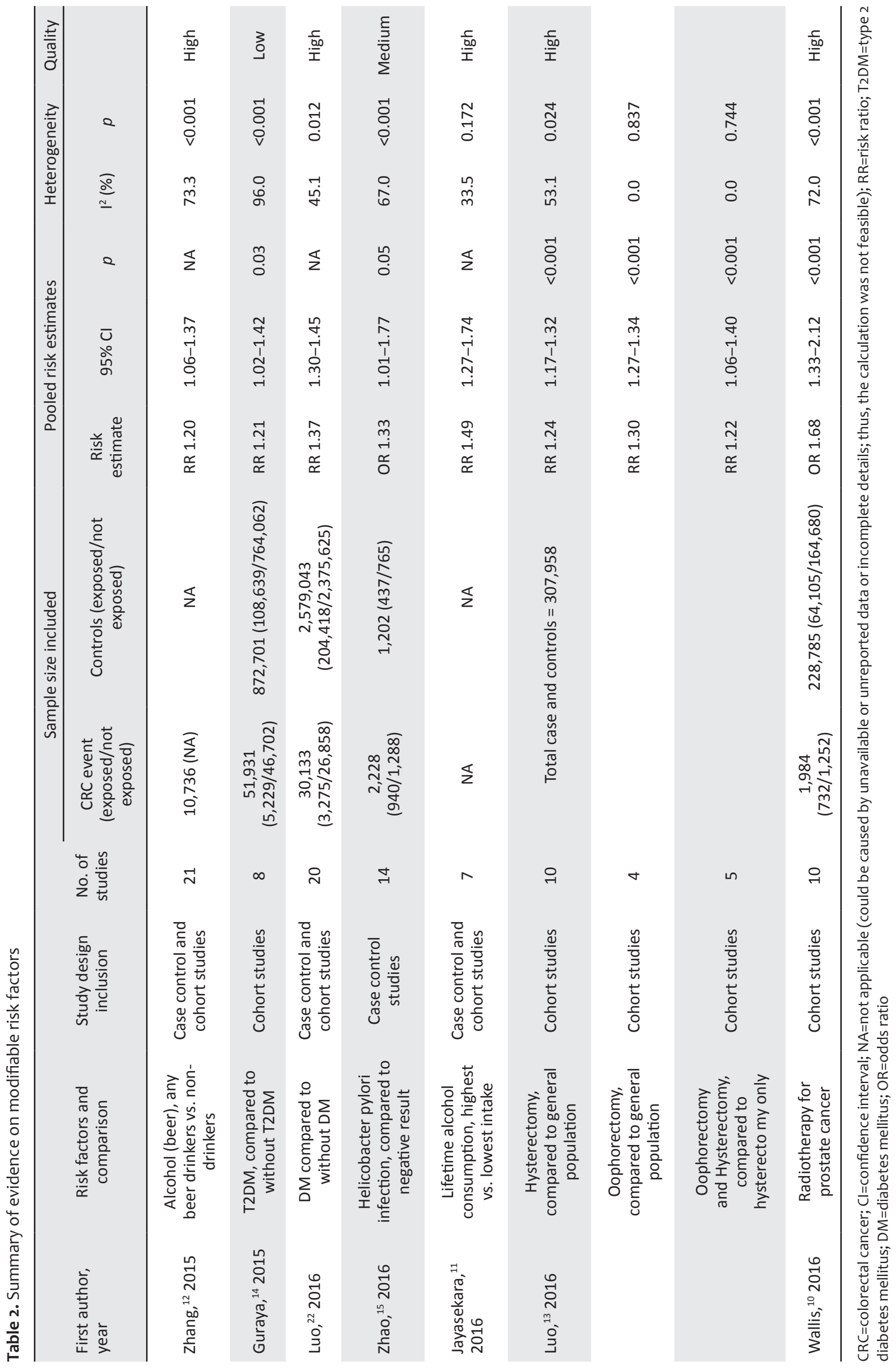




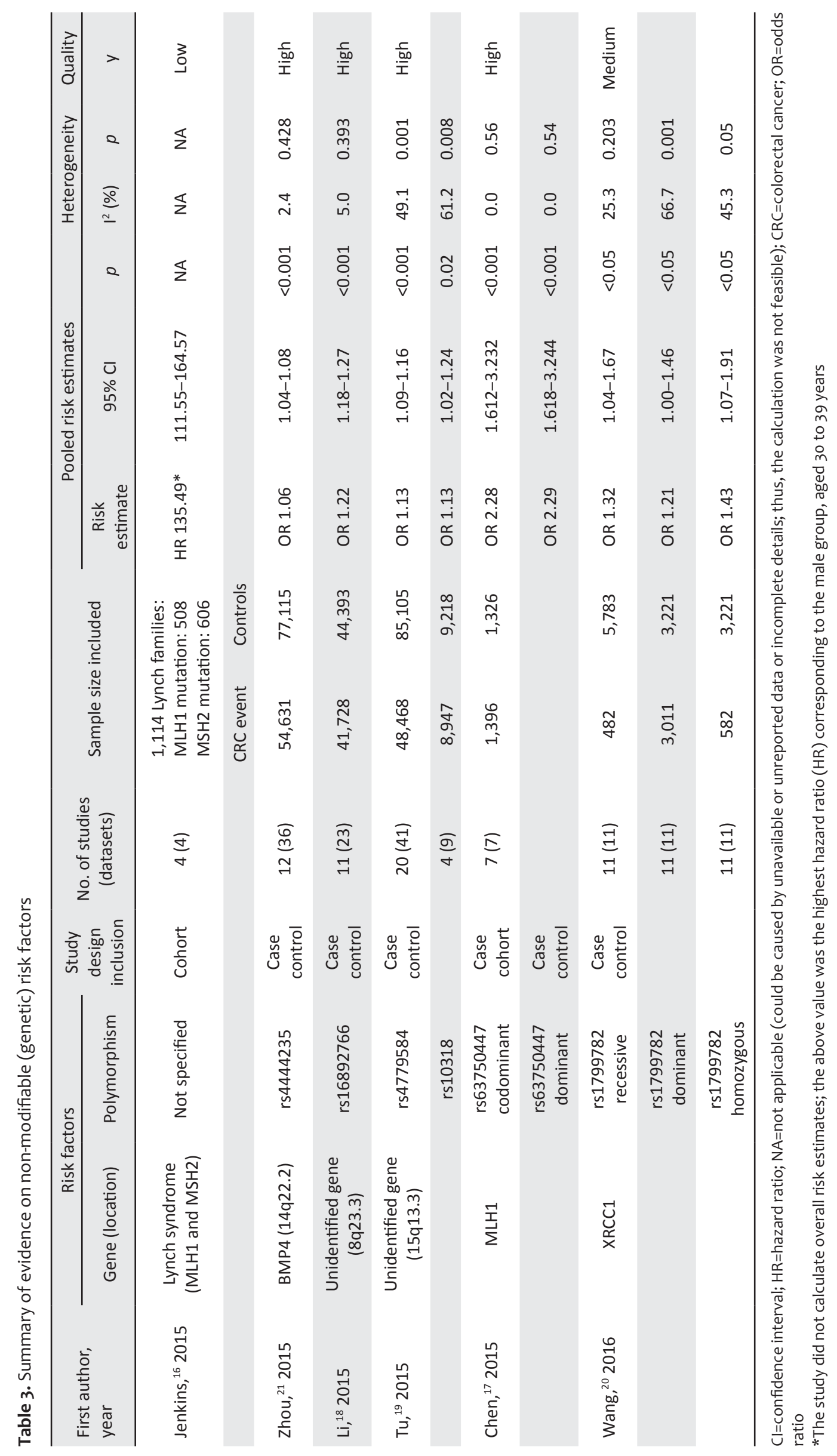


odds, which are rs1799782 in the XRCC1 gene (21-43\%) and rs16892766 in chromosome 8q23.3. One SNP in the BMP4 gene (rs4444235) only showed a small increase in CRC odds (6\% increase, $95 \% \mathrm{Cl} 4-8 \%$ ). (Table 3)

\section{DISCUSSION}

This review discovered some new and previously known risk factors for developing CRC. Radiotherapy, in any dose and duration, is associated with the future development of cancer. Wallis et $\mathrm{al}^{10}$ reported a notably increased risk, as opposed to a weak association reported by other primary research. ${ }^{23,24}$ Surgical treatment for ovarian cancer by oophorectomy increases the risk of colorectal cancer by stopping the effects of hormones. Fortunately, previous meta-analyses have shown a potential benefit of hormone replacement therapy to prevent $C R C$ in these patients. ${ }^{25}$ DM has been extensively studied and results in an increased risk not only of CRC but also of developing other cancers, such as liver and gastric cancers. ${ }^{26,27}$ This finding suggests a common cancer progenitor from digestive organs that should be studied further. Another shared digestive risk factor included in this review is $\mathrm{H}$. pylori with a similar strength of association compared to previous reviews. ${ }^{28,29}$ Alcohol consumption reviewed here is concordant with previous reviews in various populations with evidence of a dose-response relationship. ${ }^{30,31}$

The most prominent result of genetic studies is the role of mutation in Lynch syndrome (MSH2 and $\mathrm{MLH}_{1}$ ) and the future development of $\mathrm{CRC}$ from previous studies. $^{32,33}$ The increased risk for the XRCC1 and $\mathrm{BMP}_{4}$ gene mutations is comparable with a previous review. ${ }^{34,35}$ The role of a genetic mutation in the 8 q23.3 and $15 q 13.3$ loci is a novel finding that might need future research.

\section{Strength and limitations}

The strengths of this review include its systematic approach and quality assessment using the PRISMA guidelines. Given a specified range of publication dates, this review additionally included some studies with a similar theme, such as beer consumptionalcohol intake, T2DM, and the Lynch syndromeMLH1 mutation. Clear screening criteria contributed to producing a high impact meta-analysis. Some limitations should be considered when applying the results, mainly regarding the search strategy and data synthesis. The search strategy within a short time limit on one platform (MEDLINE) probably missed some informative resources. Numerous hits of studies originating from East Asia (i.e., China and Japan). These countries have a high burden of CRC and correspondingly might not have published studies in English. A search strategy that includes the Global Health and Chinese national databases could discover more publications in specific populations. The aim to find only five themes for each risk factor group based on recentness excluded some interesting risk factors with a weak association in the screening process. This may have led to selection bias, although all eligibility criteria were thoroughly described. The results of each meta-analyses should be carefully generalized as they mostly have a high level of heterogeneity.

\section{Implications for public health practice and health policy}

The modifiable risk factors discovered in this study are important for primary prevention and consideration or stratification for early detection of CRC. The high rate of secondary CRC due to radiation should inform the oncologist and radiotherapist to consider the benefit-to-harm ratio of specific localized treatment, while the gynecologic surgeon should consider the need for hormone replacement after ovary removal procedures. The evidence in diabetic patients could lead to a contradictive decision, as use of insulin poses an increased risk for developing $C R C,{ }^{36}$ while metformin therapy shows protective effects. ${ }^{37,38}$ Nevertheless, this review suggests that the health practitioner should aim for well-controlled blood glucose but does not warrant a stricter glucoselowering regimen than the current practice for T2DM treatment. The need for early CRC screening of diabetic patients requires more convincing evidence.

The evidence on Lynch syndrome would not change the current practice in high-income countries. Genetic testing is cost effective ${ }^{39}$ and many countries, including the UK have integrated genetic testing of Lynch syndrome into the high-risk group, particularly in cases of a family history of a similar disease. ${ }^{6}$ However, further information from corresponding meta-analyses regarding a decreased $\mathrm{HR}$ with increasing age should not directly decrease the screening effort. The cost-effectiveness of decreasing the screening effort according to 
age in Lynch syndrome would probably alter the recommendation. Evidence on the remaining genetic risk factors could help find the best combination of risk predictors for early detection of CRC. The focus of genetic studies in a restricted population, such as a subgroup analysis performed by some of the metaanalyses, might find a stronger association. These approaches would improve the current screening program by including important genetic markers for certain high-risk groups, as combining universal and selective screening in one multi-stage program. The pitfall would be potentially increasing false negative results.

This review supports recent efforts for managing the CRC burden in lower to middle-income countries. Particularly for genetic screening, it poses the availability and affordability issues that might hinder these countries to implement CRC screening. Current evidence on CRC burden warrants a well-designed universal or targeted screening program, with at least a simple diagnostic test, such as a stool examination, in developing countries.

Based on these findings, future research should explore the impact of treatments for pre-existing diseases, as many behavioral, metabolic, and diet risk factors have been discovered without any effects on practice. Research on the best-fit genetic marker combination is still ongoing with promising results. Another option is to combine both modifiable and non-modifiable risk factors in one study to assess any overlap and to understand the underlying mechanism of their association.

Research on risk factors for $\mathrm{CRC}$ has been widely performed, with more evidence coming from high burden developing countries. Current practices may not be changed until the discovery of novel high impact factors. In conclusion, recent evidence shows that research on CRC risk factors is continuing to grow, demonstrating that more studies on risk factors are needed to optimize CRC prevention and early detection.

\section{Conflict of Interest}

The authors affirm no conflict of interest in this study.

\section{Acknowledgment \\ None.}

\section{Funding Sources}

The author reports scholarship from Lembaga Pengelola Dana Pendidikan (LPDP) Kementerian Keuangan Republik Indonesia, during the conduct of the study.

\section{REFERENCES}

1. Ferlay J, Soerjomataram I, Dikshit R, Eser S, Mathers C, Rebelo $\mathrm{M}$, et al. Cancer incidence and mortality worldwide: sources, methods and major patterns in GLOBOCAN 2012. Int J Cancer. 2015;136(5):E359-86.

2. Douaiher J, Ravipati A, Grams B, Chowdhury S, Alatise O, Are C. Colorectal cancer-global burden, trends, and geographical variations. J Surg Oncol. 2017;115(5):619-30.

3. Global Burden of Disease Cancer Collaboration, Fitzmaurice C, Allen C, Barber RM, Barregard L, Bhutta ZA, et al. Global, regional, and national cancer incidence, mortality, years of life lost, years lived with disability, and disability-adjusted life-years for 32 cancer groups, 1990 to 2015: a systematic analysis for the global burden of disease study. JAMA Oncol. 2017;3(4):524-48.

4. Pruitt SL, Shim MJ, Mullen PD, Vernon SW, Amick BC 3rd. Association of area socioeconomic status and breast, cervical, and colorectal cancer screening: a systematic review. Cancer Epidemiol Biomarkers Prev. 2009;18(10):2579-99.

5. Wheat CL, Clark-Snustad K, Devine B, Grembowski D, Thornton TA, Ko CW. Worldwide incidence of colorectal cancer, leukemia, and lymphoma in inflammatory bowel disease: an updated systematic review and meta-analysis. Gastroenterol Res Pract. 2016;2016:1632439.

6. Cairns SR, Scholefield JH, Steele RJ, Dunlop MG, Thomas HJ, Evans GD, et al. Guidelines for colorectal cancer screening and surveillance in moderate and high risk groups (update from 2002). Gut. 2010;59(5):666-89.

7. National Guideline C. Screening for colorectal cancer: U.S. Preventive Services Task Force recommendation statement. 2016.

8. Porta M, Greenland S, Hernán M, dos Santos Silva I, Last JM. A Dictionary of Epidemiology: Oxford University Press; 2014.

9. Moher D, Liberati A, Tetzlaff J, Altman DG, PRISMA Group. Preferred reporting items for systematic reviews and meta-analyses: the PRISMA statement. PLoS Med. 2009;6(7):e1000097.

10. Wallis CJ, Mahar AL, Choo R, Herschorn S, Kodama R, Shah PS, et al. Second malignancies after radiotherapy for prostate cancer: systematic review and meta-analysis. BMJ. 2016;352:i851.

11. Jayasekara H, Maclnnis RJ, Room R, English DR. Long-term alcohol consumption and breast, upper aero-digestive tract and colorectal cancer risk: a systematic review and meta-analysis. Alcohol Alcohol. 2016;51(3):315-30.

12. Zhang C, Zhong M. Consumption of beer and colorectal cancer incidence: a meta-analysis of observational studies. Cancer Causes Control. 2015;26(4):549-60.

13. Luo G, Zhang Y, Wang L, Huang Y, Yu Q, Guo P, et al. Risk of colorectal cancer with hysterectomy and oophorectomy: a systematic review and meta-analysis. Int J Surg. 2016;34:88-95.

14. Guraya SY. Association of type 2 diabetes mellitus and the risk of colorectal cancer: a meta-analysis and systematic review. World J Gastroenterol. 2015;21(19):6026-31.

15. Zhao $\mathrm{Y}$, Wang $X$, Wang $Y$. Helicobacter pylori infection and colorectal carcinoma risk: a meta-analysis. J Cancer Research Ther. 2016;12(Supplement):15-8.

16. Jenkins MA, Dowty JG, Ait Ouakrim D, Mathews JD, Hopper $J \mathrm{~L}$, Drouet $\mathrm{Y}$, et al. Short-term risk of colorectal cancer in individuals with lynch syndrome: a meta-analysis. J Clin Oncol. 2015;33(4):326-31.

17. Chen H, Shen Z, Hu Y, Xiao Q, Bei D, Shen X, et al. Association between MutL homolog 1 polymorphisms and the risk of colorectal cancer: a meta-analysis. J Cancer Res Clin Oncol. 2015;141(12):2147-58.

18. Li M, Gu Y. Quantitative assessment of the influence of common variation rs16892766 at 8q23.3 with colorectal adenoma and cancer susceptibility. Mol Genet Genomics. 2015;290(2):461-9.

19. Tu L, Yan B, Peng Z. Common genetic variants (rs4779584 and 
rs10318) at $15 q 13.3$ contributes to colorectal adenoma and colorectal cancer susceptibility: evidence based on 22 studies. Mol Genet Genomics. 2015;290(3):901-12.

20. Wang L, Qian J, Ying C, Zhuang Y, Shang X, Xu F. X-ray crosscomplementing groups $1 \mathrm{rs} 1799782 \mathrm{C}>\mathrm{T}$ polymorphisms and colorectal cancer susceptibility: a meta-analysis based on Chinese Han population. J Cancer Res Ther. 2016;12(Supplement):C264-7.

21. Zhou L, Xie J, Gu EL, Huang Y, Qu Y, Xu AP, et al. Common genetic variant on BMP4 contributes to colorectal adenoma and cancer: a meta-analysis based on 15 studies. Cytokine. 2015;72(2):154-9.

22. Luo S, Li JY, Zhao LN, Yu T, Zhong W, Xia ZS, et al. Diabetes mellitus increases the risk of colorectal neoplasia: an updated meta-analysis. Clin Res Hepatol Gastroenterol. 2016;40(1):110-23.

23. Takam R, Bezak E, Yeoh EE. Risk of second primary cancer following prostate cancer radiotherapy: DVH analysis using the competitive risk model. Phys Med Biol. 2009;54(3):611-25.

24. Zelefsky MJ, Pei X, Teslova T, Kuk D, Magsanoc JM, Kollmeier M, et al. Secondary cancers after intensity-modulated radiotherapy, brachytherapy and radical prostatectomy for the treatment of prostate cancer: incidence and cause-specific survival outcomes according to the initial treatment intervention. BJU Int. 2012;110(11):1696-701.

25. Nanda K, Bastian LA, Hasselblad V, Simel DL. Hormone replacement therapy and the risk of colorectal cancer: a metaanalysis. Obstet Gynecol. 1999;93(5 Pt 2):880-8.

26. Yoon JM, Son KY, Eom CS, Durrance D, Park SM. Pre-existing diabetes mellitus increases the risk of gastric cancer: a metaanalysis. World J Gastroenterol. 2013;19(6):936-45.

27. Tanaka K, Tsuji I, Tamakoshi A, Matsuo K, Wakai K, Nagata C, et al. Diabetes mellitus and liver cancer risk: an evaluation based on a systematic review of epidemiologic evidence among the Japanese population. Jpn J Clin Oncol. 2014;44(10):986-99.

28. Chen YS, Xu SX, Ding YB, Huang XE, Deng B. Helicobacter pylori Infection and the risk of colorectal adenoma and adenocarcinoma: an updated meta-analysis of different testing methods. Asian Pac J Cancer Prev. 2013;14(12):7613-9.

29. Zumkeller $\mathrm{N}$, Brenner $\mathrm{H}$, Zwahlen $\mathrm{M}$, Rothenbacher $\mathrm{D}$.
Helicobacter pylori infection and colorectal cancer risk: a metaanalysis. Helicobacter. 2006;11(2):75-80.

30. Fedirko V, Tramacere I, Bagnardi V, Rota M, Scotti L, Islami F, et al. Alcohol drinking and colorectal cancer risk: an overall and dose-response meta-analysis of published studies. Ann Oncol. 2011; 22(9):1958-72.

31. Mizoue T, Inoue M, Wakai K, Nagata C, Shimazu T, Tsuji I, et al. Alcohol drinking and colorectal cancer in Japanese: a pooled analysis of results from five cohort studies. Am J Epidemiol. 2008;167(12):1397-406.

32. Li X, Yao X, Wang Y, Hu F, Wang F, Jiang L, et al. MLH1 promoter methylation frequency in colorectal cancer patients and related clinicopathological and molecular features. PLoS One. 2013;8(3):e59064

33. Wang T, Liu Y, Sima L, Shi L, Wang Z, Ni C, et al. Association between MLH1 $-93 \mathrm{G}>$ a polymorphism and risk of colorectal cancer. PLoS One. 2012;7(11):e50449.

34. Mao D, Zhang Y, Lu H, Fu X. Association between X-ray repair cross-complementing group 1 Arg194Trp polymorphism and colorectal cancer risk. Tumour Biol. 2013;34(5):2529-38.

35. Zeng FR, Ling Y, Yang J, Tian XC, Yang X, Luo RC. X-ray repair cross-complementing group $1 \mathrm{Arg} 399 \mathrm{Gln}$ gene polymorphism and susceptibility to colorectal cancer: a meta-analysis. Tumour Biol. 2013;34(1):555-63.

36. Sun A, Liu R, Sun G. Insulin therapy and risk of colorectal cancer: an updated meta-analysis of epidemiological studies. Curr Med Res Opin. 2014;30(3):423-30.

37. Liu F, Yan L, Wang Z, Lu Y, Chu Y, Li X, et al. Metformin therapy and risk of colorectal adenomas and colorectal cancer in type 2 diabetes mellitus patients: a systematic review and metaanalysis. Oncotarget. 2017;8(9):16017-26.

38. Nie Z, Zhu H, Gu M. Reduced colorectal cancer incidence in type 2 diabetic patients treated with metformin: a meta-analysis. Pharm Biol. 2016;54(11):2636-42.

39. Dinh TA, Rosner BI, Atwood JC, Boland CR, Syngal S, Vasen HF, et al. Health benefits and cost-effectiveness of primary genetic screening for Lynch syndrome in the general population. Cancer Prev Res (Phila). 2011;4(1):9-22. 